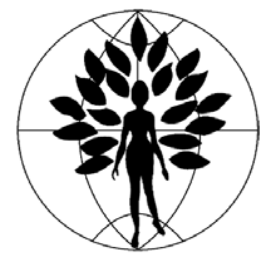

\title{
SURGERY AND TECHNOLOGY
}

\section{Miniaturizing the laparoscope: Current applications of micro- and minilaparoscopy}

\author{
Frank F. Tu ${ }^{a, *}$, A.P. Advincula ${ }^{b}$ \\ a Department of Obstetrics and Gynecology, Evanston Northwestern Healthcare, Evanston, IL, USA \\ ${ }^{\mathrm{b}}$ Department of Obstetrics and Gynecology, University of Michigan Medical Center, Ann Arbor, MI, USA
}

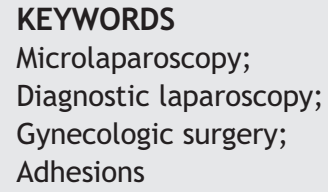

\begin{abstract}
Technological advancements have enabled miniaturization of the endoscope without significant compromise to either diagnostic or operative abilities. With a complement of instruments $<5 \mathrm{~mm}$ in diameter, newer minimally invasive techniques have been developed to decrease the pain and trauma associated with surgical procedures. These advancements involve additional options for peritoneal access as well as facilitating diagnostic evaluation of the pelvis. Although the robustness and durability of the smaller instruments is an area of concern, a thorough understanding of the clinical advantages and disadvantages has the potential to improve patient outcomes.

(c) 2007 Published by Elsevier Ireland Ltd. on behalf of International Federation of Gynecology and Obstetrics.
\end{abstract}

\section{Introduction}

From the outset, laparoscopic surgery has aimed to decrease the morbidity associated with large laparotomy incisions, and gynecologic surgeons have been at the forefront of this movement. As technology has progressed, the size of laparoscopic instruments has diminished in an attempt by surgeons to minimize the trauma induced by their procedures. One aspect of laparoscopy that has made significant strides is in the area of optics. In the past, smaller endoscopes were utilized only for visually-guided access strategies rather than for purposeful laparoscopy itself. The main reasons were that these smaller endoscopes had poor resolution, illumination, and optical clarity. Although many surgeons still prefer $10 \mathrm{~mm}$ endoscopes with the traditional rod-lens system for gynecologic laparo-

\footnotetext{
* Corresponding author. Division of Gynecological Pain and Minimally Invasive Surgery, Department of Obstetrics \& Gynecology, Evanston Northwestern Healthcare, Walgreen's Building Room 1507, 2650 Ridge Avenue, Evanston, IL 60201, USA. Tel.: +1 847570 3879; fax: +1 8475701846 .

E-mail address: Ftu@enh.org (F.F. Tu).
}

scopy, technological improvements have reduced the size of the laparoscope without significantly compromising its function. With these changes surgeons are now able to incorporate many of the potential advantages of a smaller endoscopic system and its associated instruments (Figs. 1 and 2).

The following review focuses on applications in peritoneal access and conscious pain mapping.

\section{Equipment and performance}

Prior to any discussion of clinical applications, it is important to gain an appreciation of the differences between the various laparoscopic sizes. For the purpose of this paper the term "minilaparoscope" refers to endoscopes ranging from 2-5 mm in diameter, while "microlaparoscopy" refers to endoscopes that are $<2 \mathrm{~mm}$ in diameter. Whereas the traditional $10 \mathrm{~mm}$ as well as some $5 \mathrm{~mm}$ laparoscopes rely on a rod-lens system, today, the smaller, newer-generation endoscopes incorporate semi-rigid and deflectable fiberoptic bundles, which have allowed successful reduction in size to less than $5 \mathrm{~mm}$. 


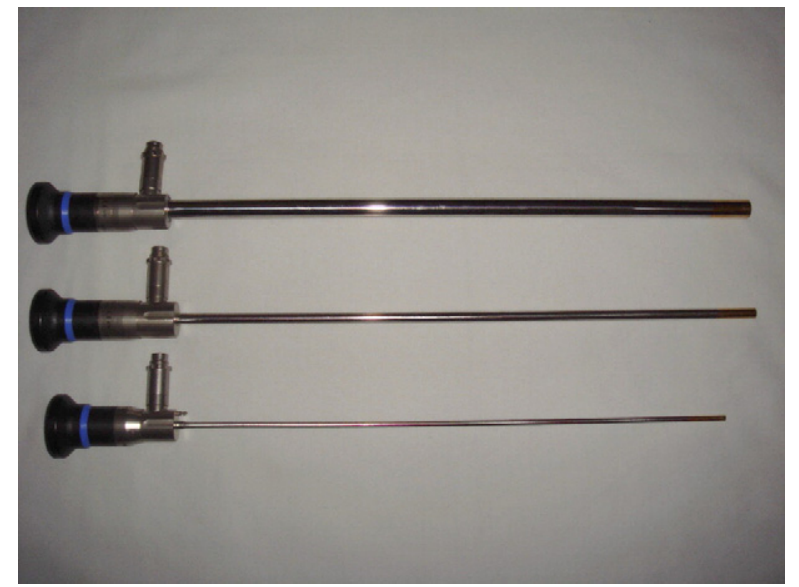

Figure 1 Photograph, from top to bottom, comparing $10 \mathrm{~mm}$, $5 \mathrm{~mm}$, and $3 \mathrm{~mm} 0^{\circ}$ laparoscopes.

Although today's miniaturized laparoscopes are an improvement over their predecessors, small size makes them fragile and they still have a lower resolution and decreased depth of field compared with their larger rod-lens counterparts. Another significant difference is that the smaller fiberoptic-based laparoscopes only come in $0^{\circ}$ lenses whereas those with rod-lens systems can provide various degrees of view $\left(30^{\circ}, 45^{\circ}, 70^{\circ}\right)$.

An understanding of the limitations of this miniaturized equipment is essential when incorporating this technology into your surgical practice. One major concern involves the ability to adequately visualize the diagnostic region of interest or to perform a required surgical task as effectively as one can with larger laparoscopes. Faber and Doddington [1] were able to show equivalence in diagnostic accuracy in 52 patients when comparing a $2 \mathrm{~mm}$ microlaparoscope with a $10 \mathrm{~mm}$ standard laparoscope. However, the authors did note that more complicated surgery with the smaller laparoscope would be hampered by the limited field of view. This was further supported by Rosser et al. [2] in a study of 60 experienced laparoscopists with advanced operative skills. They concluded that even for a skilled laparoscopic surgeon, the use of microlaparoscopes impairs performance and requires significantly higher skills than those needed in traditional operative environments. Despite these limitations, Kovacs et al. [3] studied 135 patients and strongly advocated the use of the microlaparoscope as the instrument of choice for initial diagnostic laparoscopy. They noted less postoperative abdominal pain and decreased analgesic requirements.

As with miniaturized endoscopes, complementary instruments ( $\leq 2 \mathrm{~mm}$ in diameter) have become increasingly available. A variety of instruments exist that allow the performance of basic surgical tasks such as grasping, dissecting, suturing, aspiration, and even electrosurgery. Similar to the smaller endoscopes, durability remains a significant concern and care must be exercised in the handling and use of these instruments.

\section{Peritoneal access}

Perhaps the most valuable skill to learn when utilizing either micro- or minilaparoscopy is facilitating the left upper quad- rant technique for initial abdominal entry. Women with a history of multiple surgeries, pregnancy, large pelvic masses, an umbilical hernia, and/or known intestinal adhesions beneath the umbilicus can be at risk for failed entry through the umbilicus. The use of the left upper abdominal quadrant as an alternate site for initial trocar placement and peritoneal access can potentially avoid serious morbidity. Initially placing the camera high in the abdomen enables the surgeon to obtain a clear view of the lower abdomen and pelvis without being hampered by the viscera directly in the lens face, or being surrounded by a wall of intestinal adhesions. Indeed, for large leiomyomatous uteri or adnexal cysts, the choice of the umbilicus for primary camera placement may not be ideal at any point in the case.

Techniques described to perform left upper quadrant entry include either Veress needle insufflation at Palmer's point (along the mid-clavicular line, $3 \mathrm{~cm}$ below the left costal margin) or direct cannula insertion at the same site [4,5] (Fig. 3). Pre-procedural insertion of an orogastric tube is useful to avoid inadvertent puncture of a distended stomach at this site. Although this procedure should be straightforward, several key tips can ensure success. First, use anesthesia to help confirm selection of the correct site because the intra-operative view of the clavicle will be limited by the sterile drapes. A $5 \mathrm{~mm}$ or smaller incision can be made at this site depending on the size of the telescope available. Secondly, dissect the pre-fascial tissue with a Kelly clamp to smooth the passage of the Veress needle. Pinching the abdominal wall between your fingers at the site of entry should allow an estimate of how much abdominal wall needs to be traversed by the Veress needle to avoid excessive past-pointing and inadvertent omental tears. To insert the Veress in the right plane and avoid pre-peritoneal insufflation, set the tip against the fascia prior to lifting the abdominal wall up, aiming down towards the pelvis in the same line as the midclavicular line. The Veress needle should be angled at $45^{\circ}$ relative to the plane of the abdominal wall. Lift the wall up and feel the Veress needle traverse the fascial layer and the peritoneum. Proceed to check proper position within the low pressure environment of the peritoneal cavity before insufflating and inserting the initial port. Reported results with this approach are limited; however, in 2 retrospective series 81 patients at risk for bowel adhesions were successfully

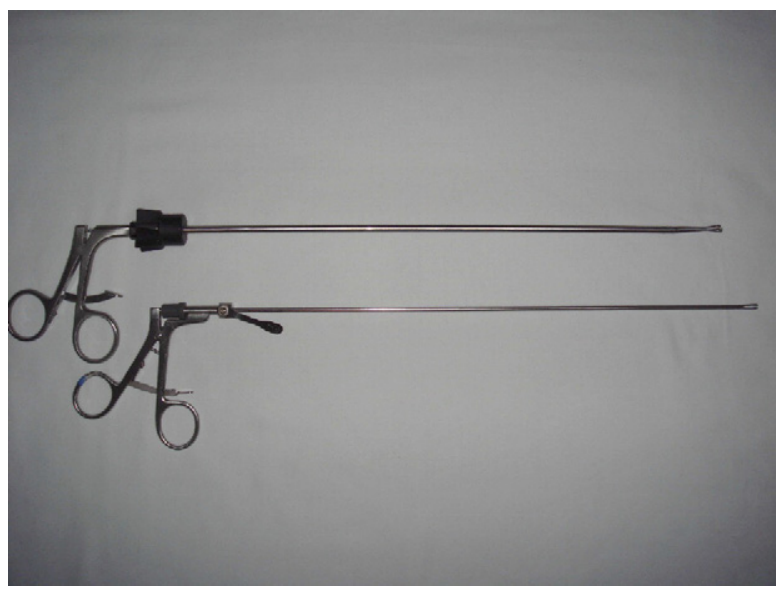

Figure 2 Photograph comparing $5 \mathrm{~mm}$ and $3 \mathrm{~mm}$ instruments. 


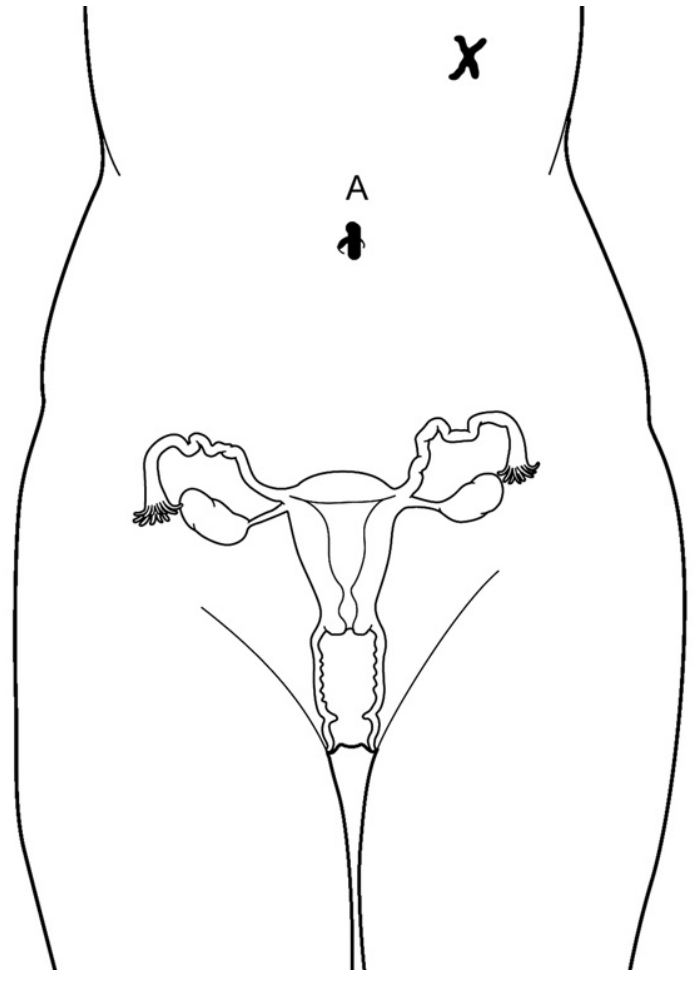

Figure 3 Schematic of Palmer's point during left upper quadrant entry. Umbilicus (A) and Palmer's point (X).

entered using this approach, with no complications directly related to cannula insertion $[5,6]$. In the largest series published by Audebert and Gomel [7], a $1.2 \mathrm{~mm}$ microlaparoscope was used for access in 814 consecutive patients without major access injury for an overall success rate of $99.8 \%$. Using a small incision to allow initial access by this route poses minimal risk to the patient, yet the visualization provided by a microlaparoscope from this vantage point is a key resource in determining how to proceed in difficult operating environments. Once an overall perspective of the intra-abdominal topography is gained, additional ports can be placed under direct visualization to assist in taking down any interfering adhesions and, if necessary, to clear the umbilicus for port placement. Using a smaller endoscope and instruments enables additional visibility through any of the 4 quadrants of the abdomen, or even transvaginally through the cul-de-sac, to restore many key perspectives inherently lost in the constrained laparoscopic operating environment.

\section{Conscious pain mapping}

\subsection{Background}

One of the most exciting surgical applications of the miniaturized endoscope is to perform an examination of the pelvis under local anesthesia. Palter and Olive [8] first described office-based microlaparoscopy under conscious sedation in 1996, a procedure that became known as conscious pain mapping. In 1925, Short [9] described diagnostic celioscopy under local anesthesia to evaluate the liver. The smaller instrumentation of the modern era enables a much more complicated evaluation. With conscious pain mapping, women receive sufficient analgesia and sedatives to allow comfortable placement of small ( $3-5 \mathrm{~mm}$ ) ports and instruments. Subsequently, they can be awakened intraoperatively to consciously report the effects of traction and palpation of intra-abdominal structures in real time. Bypassing the potential pain generators in the anterior abdominal wall (such as trigger points) allows the gynecologist to specifically evaluate for palpation-induced visceral sensitivity and to document the presence of any anatomic pathology. In theory this approach allows for more selective removal of only demonstrably tender tissue. Other potential advantages include decreased surgical risk by avoidance of general anesthesia and quicker recovery due to the significantly diminished tissue trauma.

\subsection{Technique}

Careful patient selection is critical; contraindications include a high-risk anesthetic profile (higher than American Society of Anesthesiologists [ASA] Class II), obesity, strong preoperative suspicion of severe intra-abdominal adhesions, or history of psychiatric disorders. This procedure can be performed in the operating room or in an office setting [8]. Prior to the procedure, the patient should be counseled thoroughly on the conditions to expect. She should be advised that pain mapping will stop at any time the discomfort becomes excessive, and that she will then receive adequate pain relief and/or general anesthesia. The use of a standardized pain assessment instrument, such as a verbal 10-point scale for elicited pain intensity, should be reviewed with the patient prior to the start of the surgery.

Intra-operatively, the procedure begins by orienting the patient in the Trendelenburg position before administering any sedation. Low-dose fentanyl $(50 \mu \mathrm{g})$ given intermittently during the procedure typically gives adequate baseline pain relief. Propofol can be given to provide a deeper level of sedation during initial trocar placement. Dexmedetomidine, an $\alpha-2$ agonist, can be titrated to provide readily reversible sedation and analgesia. Generous umbilical and suprapubic field blocks through the entire thickness of the abdominal wall are critical to avoid confounding of the results by abdominal wall irritation; the use of $1 \%$ lidocaine buffered 9:1 with $0.9 \%$ bicarbonate is an example of one regimen. During conscious pain mapping the use of trocars $5 \mathrm{~mm}$ or smaller is essential for patient comfort. Lidocaine gel should be used to anesthetize the urethra prior to catheterizing the bladder. If desired, a paracervical block will allow comfortable insertion of a uterine manipulator; however, use of the block may limit the mapping by anesthetizing the uterus. An alternative is the introduction of an expandable fan retractor which can be used to gently elevate the uterus through a lateral port. To minimize patient discomfort from carbon dioxide irritation it has been suggested that the surgeon observe a total volume limit for carbon dioxide of $1 \mathrm{~L}$ and intra-abdominal pressures of $10 \mathrm{~mm} \mathrm{Hg}$ [10].

Once the trocars are in place, begin by jostling them gently to ensure that abdominal wall anesthesia is sufficient. The current 2-5 $\mathrm{mm}$ range instruments and endoscopes should be sufficient for visualization and manipulation. Pain mapping is then conducted in a systematic fashion, ideally with an assistant recording elicited pain scores on a preprinted drawing of the pelvis. The surgeon must move deliberately to maximize 
patient tolerance and comfort. Using a blunt probe through a suprapubic or lateral port, palpation usually begins with an insensitive structure such as the small intestine or rectosigmoid to establish a baseline response to manipulation. The bowels can be retracted with gentle nudging and the Trendelenburg position to rest above the pelvic brim, allowing visualization of the reproductive viscera.

The examination should proceed sequentially, encompassing the broad ligaments, the uterine ligaments, the adnexae with associated fossa, the uterus, both cul-de-sacs, and the appendix. Querying the patient for pain scores at each point of palpation should only take about 5-10 min in total. An attempt should be made to determine if the pain specifically resembles the symptoms afflicting the patient. Adhesions and visible peritoneal lesions should also be palpated. The choice to allow patients to observe the surgery on a monitor is controversial; some bias may be introduced by patients who have firm convictions that their pain is clearly of a visceral origin. Hence many surgeons limit the patient's view of the procedure. Once the pain mapping is complete, a decision should be made whether any relevant tissues should be biopsied or removed. The exact extent of the surgery should be agreed upon with the patient preoperatively if the pain mapping is to be converted to an operative laparoscopy. This will often necessitate the use of general anesthesia.

An interesting modification to the aforementioned technique was published by Steege [11] as a way to possibly aid distinguishing peripheral causes of pain from central pain syndromes. Two cases were described in which an intraoperative superior hypogastric plexus block was performed during the pain mapping. Lidocaine was injected beneath the peritoneum overlying the sacral promontory and the pain mapping repeated after several minutes to determine if a presacral neurectomy was appropriate.

\section{Results}

In several published series the success rates for completion of pain mapping ranged from $70 \%-100 \%$. Findings often included tender endometriosis implants and adhesions, focal visceral discomfort of the reproductive viscera or bladder, and generalized visceral sensitivity of the peritoneal lining. Postoperative improvement has ranged from $44 \%-94 \%$ in the different series $[8,12,13]$. However, without the benefit of randomized controlled trials, caution should be undertaken prior to making any conclusions about the absolute benefit of this diagnostic approach based solely on the small case series previously reported. Regardless, Zupi et al. [14] demonstrated that minilaparoscopy under conscious sedation for pain mapping in 20 infertile women without pain or pathology revealed consistently negative findings, thereby validating the value of this clinical measurement. Additionally, Demco [15] evaluated pain referral patterns in the pelvis and noted pain mapping as excellent in correlating the symptom with the pathology.

Despite the known limitations of the miniaturized endoscopes and their associated instruments, several studies have demonstrated the ability to not only diagnose pathology but also treat it by utilizing the same conscious sedation regimen. In a published series, Almeida et al. [16] performed successful diagnostic microlaparoscopy in 74 consecutive patients followed by successful therapeutic microlaparoscopy in 52 patients (70.2\%) utilizing the same anesthetic protocol. Finally, the financial impact resulting from the ability to perform office microlaparoscopy under local anesthesia (OLULA) was noted by Palter and Olive [8]. They concluded that OLULA was associated with an almost $80 \%$ reduction in billed charges, averaging US $\$ 1700$ versus US $\$ 7500$ for operating room laparoscopy.

\section{Conclusion}

As technology continues to evolve, gynecologic surgeons are able to take advancements in instrumentation and further impact clinical practice and outcomes in a field that is already well versed in minimally invasive techniques. The endoscope is a great example of where refinements in laparoscopy have led to additional options and improvements in how the peritoneal cavity is accessed. In the quest to increase the safety and minimize the trauma and pain involved with surgery, a method for evaluating chronic pelvic pain and performing therapeutic endoscopy under conscious sedation was also developed. Reports are now surfacing of applications such as the microlaparoscopy-assisted vaginal hysterectomy [17]. Most recently, Tsin et al. [18] described the use of miniaturized endoscopes in minilaparoscopy-assisted natural orifice surgery (MANOS). Overall, as durability is increased in these smaller instruments and optical quality further refined, the applications of minimally invasive gynecologic surgery will continue to grow.

\section{References}

[1] Faber BM, Coddington III CC. Microlaparoscopy: a comparative study of diagnostic accuracy. Fertil Steril 1997;67:952-4.

[2] Rosser JC, Olive DL, Zreik T, Duleba A, Arici A, Rutherford T, et al. Decreased performance of skilled laparoscopic surgeons at microlaparoscopy versus traditional laparoscopy. J Am Assoc Gynecol Laparosc 1996;3(Suppl 4):S44.

[3] Kovacs GT, Baker G, Dillon M, Peters M. The microlaparoscope should be used routinely for diagnostic laparoscopy. Fertil Steril 1998;70:698-701.

[4] Palmer R. Safety in laparoscopy. J Reprod Med 1974;13:1-5.

[5] Howard FM, El-Minawi AM, DeLoach VE. Direct laparoscopic cannula insertion at the left upper quadrant. J Am Assoc Gynecol Laparosc 1997;4:595-600.

[6] Tulikangas PK, Robinson DS, Falcone T. Left upper quadrant cannula insertion. Fertil Steril 2003;79:411-2.

[7] Audebert AJ, Gomel V. Role of microlaparoscopy in the diagnosis of peritoneal and visceral adhesions and in the prevention of bowel injury associated with blind trocar insertion. Fertil Steril 2000;73:631-5.

[8] Palter SF, Olive DL. Office microlaparoscopy under local anesthesia for chronic pelvic pain. J Am Assoc Gynecol Laparosc 1996;3:359-64.

[9] Short AR. The uses of celioscopy. BMY 1925;2:254-5.

[10] Howard FM. The role of laparoscopy as a diagnostic tool in chronic pelvic pain. Baillieres Best Prac Res Clin Obstet Gynaecol 2000;14:467-94.

[11] Steege JF. Superior hypogastric block during microlaparoscopic pain mapping. J Am Assoc Gynecol Laparosc 1998;5:265-7.

[12] Howard FM, El-Minawi AM, Sanchez RA. Conscious pain mapping by laparoscopy in women with chronic pelvic pain. Obstet Gynecol 2000;96:934-9.

[13] Almeida OD, Val-Gallas JM. Conscious pain mapping. J Am Assoc Gynecol Laparosc 1997;4:587-90.

[14] Zupi E, Sbracia M, Marconi D, Zullo F, Santi K, Solima E, Romanini $C$. Pain mapping during minilaparoscopy in infertile 
patients without pathology. J Am Assoc Gynecol Laparosc 1999;6:51-4.

[15] Demco LA. Pain referral patterns in the pelvis. J Am Assoc Gynecol Laparosc 2000;7:181-3.

[16] Almeida OD, Val-Gallas JM, Browning JL. A protocol for conscious sedation in microlaparoscopy. J Am Assoc Gynecol Laparosc 1997;4:591-4.
[17] Almeida OD. Microlaparoscopic-assisted vaginal hysterectomy in the morbidly obese patient. JSLS 2004;8:229-33.

[18] Tsin DA, Colombero LT, Lambeck J, Manolas P. Minilaparoscopyassisted natural orifice surgery. JSLS 2007;11:24-9. 